\title{
Nonlinear Torsional Vibration Modeling and Characteristic Study of Planetary Gear Train Processing Device
}

\author{
Sun Zhijun, Hou Li, Chang Qinglin and Wei Yongqiao \\ School of Manufacturing Science and Engineering, Sichuan University, Chengdu 610065, China
}

\section{Li Wei}

School of Mechanical Engineering \& Automation, Xihua University, Chengdu 610039, China

(Received 19 December 2013; accepted 20 May 2014)

\begin{abstract}
A nonlinear torsional vibration model with meshing errors, time varying meshing stiffness, damping coefficients, and gear backlashes was presented to analyse the nonlinear dynamic behaviour of the planetary gear train system, which was used to machine the Circular-Arc-Tooth-Trace cylindrical gear. Its dimensionless equations of the system were derived, and the solution of the equations was carried out by using the method of numerical integration. The bifurcation diagrams indicated that the system had abundant bifurcation properties with the dimensionless speed, and the damping ratios of meshing pairs could influence the vibration amplitudes and bifurcation characteristic greatly. The phase plane plots and Poincar maps revealed that the motion state of the system would through the regions such as harmonic response, non-harmonic response, 2T-periodic harmonic response, 4T-periodic harmonic response, quasi-harmonic response, and chaotic response. The chaotic regions will cause the system failure and instabilities, so these regions should be avoided.
\end{abstract}

\section{INTRODUCTION}

The planetary gear was used in the processing device for machining the Circular-Arc-Tooth-Trace cylindrical gear (CATT gear-it is a new type gear) due to its advantages, such as compactness, large torque-to-weight ratio, large transmission ratios, reduced vibrations, and translational property. ${ }^{1}$ The processing device consists of planetary gear sets that have translational and rotary motions, which can form the ideal tooth profile of the CATT gear, ${ }^{2}$ and its vibration influences the correct manufacturing of the tooth profile. That is the reason why this paper focuses on the vibration of the planetary gear transmission system. The structures of the processing device of the CATT gear are shown in Figs. 1 and 2. Similarly, there have been numerous studies about the vibration of planetary gears in recent decades. The factors influencing the vibrationand noise-related dynamic responses of planetary gear systems have been investigated by many researchers. Velex and Flamand, ${ }^{3}$ Kahraman and Blankenship, ${ }^{4}$ and Lin and Parker ${ }^{5}$ investigated the time-varying mesh stiffness. Kahraman, Parker, et al. analysed the natural modes of planetary gears with unequally spaced planets and an elastic ring gear. ${ }^{6,7}$ Ericson and Parker ${ }^{8,9}$ investigated the effects of torque on the dynamic behavior and system parameters of planetary gears by experimental measurement and finite element analysis, and the study provided good methods for the CATT gear research. The transmission errors, the spacing, and backlash-related nonlinear dynamics were the main focus in much published research. ${ }^{10-12}$ Xihui, Liang, et al. ${ }^{13}$ investigated the vibration properties of a planetary gear set and evaluated the mesh stiffness effectively. $\mathrm{Li}, \mathrm{Wu}$, and $\mathrm{Zhang}^{14}$ formulated a nonlinear time-varying dy- namic model for a multi-stage planetary gear train. However, these published studies investigated the vibration based on the conventional planetary gears. Fewer studies are available about the investigations on the translational planetary gear train.

Although many models in previous research are different from this planetary gear set, some studies can provide many available methods, as in some of the work referenced in this paper. A. Kahraman used a family of torsional dynamic models of compound gear sets to predict the free vibration characteristics under different kinematic configurations resulting in different speed ratios, but he investigated the planetary gear sets without nonlinear models. ${ }^{15}$ Robert G. Parker examined the effectiveness of planet phasing to suppress planetary gear vibration in certain harmonics of the mesh frequency based on the physical forces acting at the sun-planet and ring-planet meshes. ${ }^{16}$ This research proposed a method to suppress the vibration of the planetary gears. J. Lin and R. G. Parker also investigated the natural frequency and vibration mode sensitivities to system parameters for both tuned and mistuned planetary gears. ${ }^{17,18}$ V. K. Ambarisha, et al. investigated the complex, nonlinear dynamic behaviour of spur planetary gears using two models: a lumped-parameter model and a finite element model. ${ }^{19}$ In this paper, mesh phasing rules to suppress rotational and translational vibrations in planetary gears were valid even when nonlinearity formed tooth contact loss occurs. Sun Zhimin, et al. used a clearance-type nonlinear dynamic model of a $2 \mathrm{~K}-\mathrm{H}$ planetary gear train to analyse the nonlinear dynamic behaviour of the gear train excited by a static transmission error in addition to a mean torque. ${ }^{20}$ His research results indicate that the backlash induces complicated nonlinear dynamic behaviour in the $2 \mathrm{~K}-\mathrm{H}$ planetary gear train. Simi- 


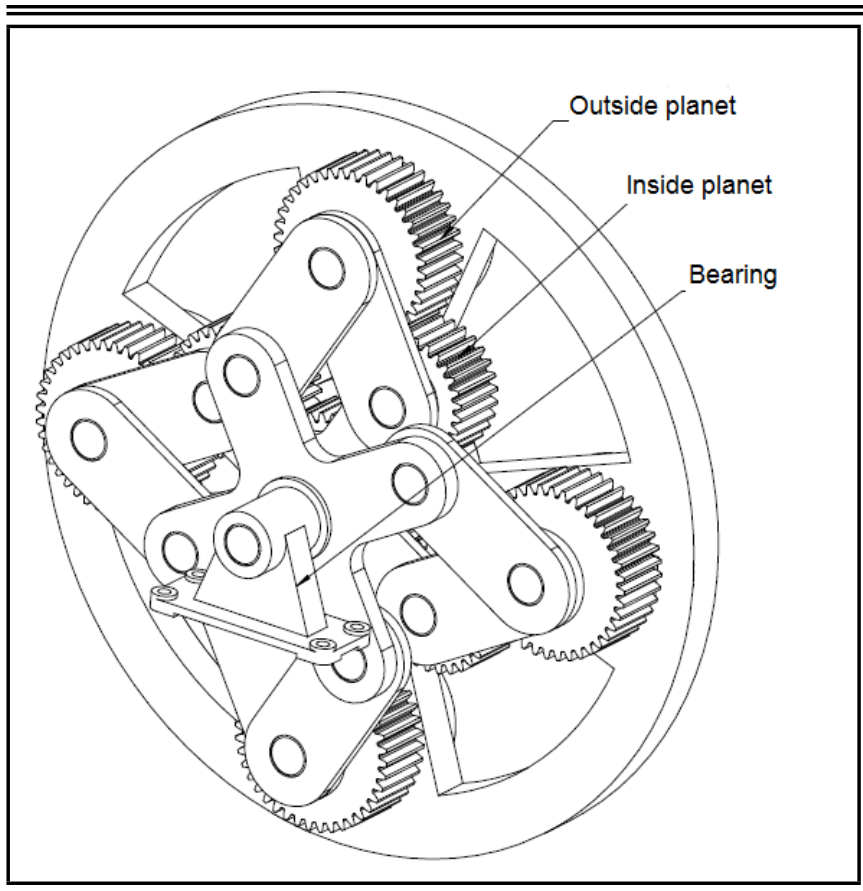

Figure 1. 3D model of the CATT gear processing device.

larly, Li Tongjie, et al. established a nonlinear torsional vibration model of a planetary gear train with errors of transmission, time varying stiffness, and gear backlashes. His study results revealed that the systems motion state would change into chaos in the way of crisis as speed increased, and a smaller damping coefficient would make the systems periodic motion state change into a complex state. ${ }^{21}$

This study proposes a planetary gears device whose planetary gear centre could move with translational motion. Then, a nonlinear torsional vibration model of the planetary gear train is established. This model includes the transmission, time varying meshing stiffness, and gear backlashes. By using the method of numerical integration, the frequency content and geometry of the dynamic response of spur planetary gears in the rotating and stationary reference frames are investigated. This paper tries to improve the stability of the planetary gear train processing device by examining the vibration characteristics of the model.

\section{MODELLING METHODOLOGY}

The planetary gear train processing device consists of a sun gear (s), $\mathrm{N}$ inside planetary gears (q), $\mathrm{N}$ outside planetary gears (p), and a carrier (c) without a ring gear. Figures 1 and 2 are the 3D models of the CATT gear processing device with four gear sets, and these gear sets have to be evenly distributed around the sun gear. All gears are spur gears, and the motion of the sun gear is constrained. Each element has one rotational degree-offreedom without considering translations.

The planetary gear dynamic model used is based on the one developed by Lin and Parker. ${ }^{5,18}$ But the structure and the motions of the model are different from those in previous research. According to the motion properties of the planetary gear device, the vibration system can be a simplified spring-damping vibration system with meshing errors and gear backlashes. The torsional vibration model is shown in Fig. 3.

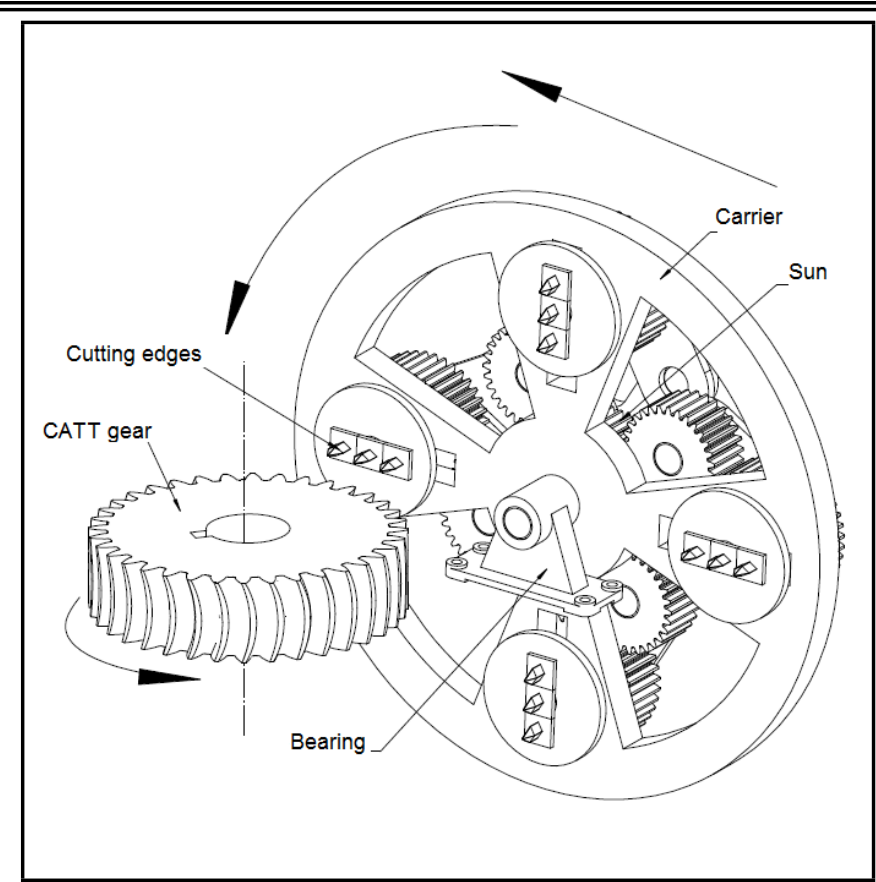

Figure 2. The CATT gear and its planetary gear train processing device.

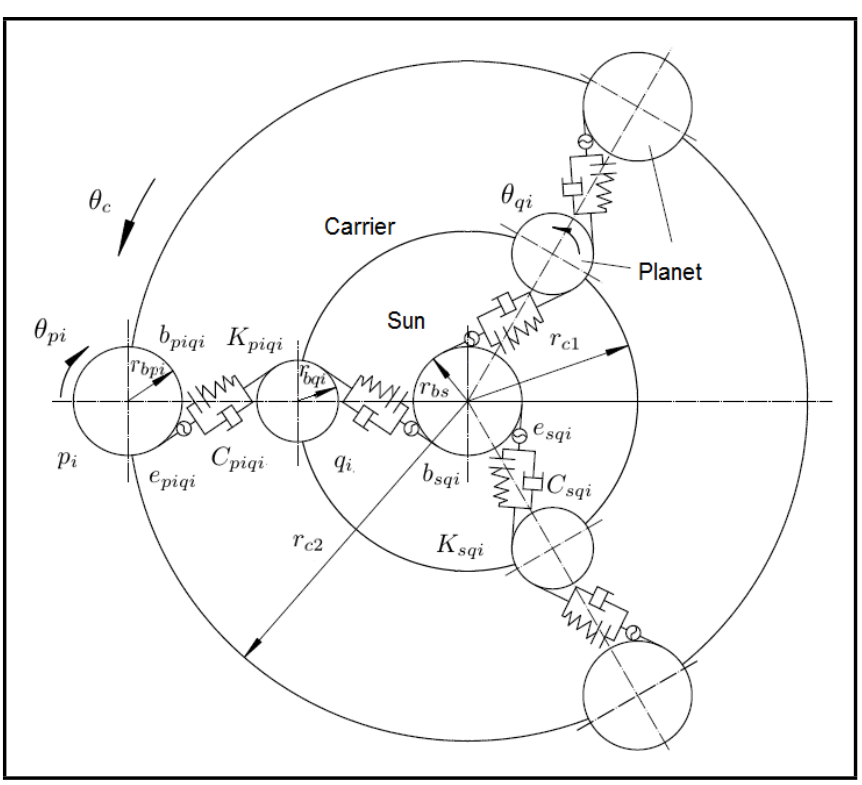

Figure 3. Torsional vibration model of planetary gears.

The model is normally selected with three or four gear sets for vibration analysis. Rotational motions of the carrier, inside planets, and outside planets are denoted by $\theta_{h}, h=c, q, p$, and $1 \ldots N$, where $N$ indicates the number of planets. The gear bodies are assumed to be rigid with moments of inertia $J_{c}, J_{q i}, J_{p i}(i=1,2, \ldots, N)$. Not only the sun and inside planet tooth meshes, but also the inside planet and the outside planet tooth meshes, are modelled as linear springs with time-varying stiffness $K_{s q i}(t), K_{\text {piqi }}(t)(i=1,2, \ldots, N)$. Simultaneously, the non-linear factors such as the damping $C_{s q i}, C_{p i q i}$, clearance $b_{s q i}, b_{p i q i}$, and meshing error $e_{s q i}(t), e_{p i q i}(t)$ are considered in the determination of instability boundaries. By using 
the Lagrange equation, the systems equations of motion are

$$
\left\{\begin{array}{l}
J_{q i} \ddot{\theta}_{q i}-\left(D_{s q i}+P_{s q i}-D_{p i q i}-P_{p i q i}\right) r_{b q i}=0 \\
J_{p i} \ddot{\theta}_{p i}+T_{L 1}=\left(P_{p i q i}+D_{p i q i}\right) r_{b p i} \\
\left(J_{c}+\sum_{i=1}^{N}\left(m_{p i} r_{c 2}+m_{q i} r_{c 1}\right)\right) \ddot{\theta}_{c}+T_{L 2} \\
+\sum_{i=1}^{N}\left(D_{s q i}+P_{s q i}\right) r_{b s} \cos \alpha=T_{D}
\end{array}\right.
$$

where $r_{b s}, r_{b q i}, r_{b p i}(i=1,2, N)$ are the base circle radii of the sun, inside planets and outside planets, respectively. The parameters $r_{c 1}$ and $r_{c 2}$ are the radii of the circle passing through the planets centres for the carrier. $m_{s}, m_{q i}, m_{p i}, m_{c}$ are the masses of the sun gear, inside planet, outside planet, and carrier. $T_{d}, T_{L 1}, T_{L 2}$ are external torques. $P_{s q i}, P_{\text {piqi }}(i=$ $1,2, \ldots, N)$ are the elastic meshing forces, and $D_{\text {sqi }}, D_{\text {piqi }}(i=$ $1,2, \ldots, N)$ are the viscous meshing forces. ${ }^{16}$ They can be expressed as

$$
\begin{gathered}
\left\{\begin{array}{l}
P_{s q i}=K_{s q i}(t) f\left(\theta_{c} r_{c 1}-\theta_{q i} r_{b q i}-e_{s q i}(t), b_{s q i}\right) \\
P_{p i q i}=K_{p i q i}(t) f\left(\theta_{q i} r_{b q i}-\theta_{p i} r_{b p i}-e_{p i q i}(t), b_{p i q i}\right)
\end{array}\right. \\
\left\{\begin{array}{l}
D_{s q i}=C_{s q i}\left(\dot{\theta}_{c} r_{c 1}-\dot{\theta}_{q i} r_{b q i}-\dot{e}_{s q i}(t)\right) \\
D_{p i q i}=C_{p i q i}\left(\dot{\theta}_{q i} r_{b q i}-\dot{\theta}_{p i} r_{b p i}-\dot{e}_{p i q i}(t)\right)
\end{array}\right.
\end{gathered}
$$

where $f(x, b)$ is the nonlinear function of clearance, and it can be represented by ${ }^{22}$

$$
f(x, b)=\left\{\begin{array}{l}
x-b, \quad(x>b) \\
0, \quad(-b \leq x \leq b) \\
x+b, \quad(x<-b)
\end{array} .\right.
$$

For spur gears, rectangular waves are often used to approximate mesh stiffness alternating between $n$ and $n+1$ pairs of teeth in comtact. ${ }^{5}$ Each mesh stiffness is represented by

$$
\left\{\begin{array}{l}
K_{s q i}(t)=K_{m s q i}+K_{a s q i} \sin \left(\omega t+\varphi_{s q i}\right) \\
K_{\text {piqi }}(t)=K_{m p i q i}+K_{a p i q i} \sin \left(\omega t+\varphi_{\text {piqi }}\right)
\end{array}\right.
$$

where $K_{m s q i}, K_{m p i q i}(i=1,2, \ldots, N)$ are mean values, and $K_{\text {asqi }}, K_{\text {apiqi }}(i=1,2, \ldots, N)$ are time-varying components of $i$ th the sun to inside planet, and inside planet to outside planet meshes. $\omega$ is the mesh frequency of the sun-planet, and $\varphi_{\text {sqi }}, \varphi_{\text {piqi }}(i=1,2, \ldots, N)$ are the phases.

$C_{s q i}, C_{p i q i}$ are the damping coefficients, and they can be expressed as ${ }^{20}$

$$
\left\{\begin{array}{l}
C_{s q i}=2 \xi_{1} \sqrt{K_{m s q i} /\left(1 / M_{s}+1 / M_{q i}\right)} \\
C_{p i q i}=2 \xi_{2} \sqrt{K_{m p i q i} /\left(1 / M_{p i}+1 / M_{q i}\right)}
\end{array} ;\right.
$$

where $\xi_{1}, \xi_{2}$ are the damping ratios of meshing pairs for the sun to inside planet, and the inside planet to outside planet. $M_{c}, M_{q i}, M_{p i}, M_{s}$ are the equivalent masses of the sun, planets, and carrier. Their expressions will be written in the following part of the paper. $e_{s q i}(t), e_{\text {piqi }}(t)$ are the gear backlashes, and can be represented by ${ }^{20}$

$$
\left\{\begin{array}{l}
e_{s q i}(t)=E_{s q i} \sin \left(\omega t+\phi_{s q i}\right) \\
e_{p i q i}(t)=E_{p i q i} \sin \left(\omega t+\phi_{p i q i}\right)
\end{array} ;\right.
$$

where $E_{s p i}, E_{\text {piqi }}$ are the synthetical meshing errors, and $\phi_{\text {spi }}, \phi_{\text {piqi }}$ are the phase angles.
In order to eliminate the displacement of the rigid body, the generalized coordinates are introduced as

$$
\left\{\begin{array}{l}
X_{s q i}=x_{c}-x_{q i}-e_{s q i}(t) \\
X_{p i q i}=x_{q i}-x_{p i}-e_{p i q i}(t)
\end{array} .\right.
$$

Eq. (8) can also be expressed as

$$
\left\{\begin{array}{l}
X_{s q i}=\theta_{c} r_{c 1}-\theta_{q i} r_{b q i}-e_{s q i}(t) \\
X_{p i q i}=\theta_{q i} r_{b q i}-\theta_{p i} r_{b p i}-e_{p i q i}(t)
\end{array} .\right.
$$

Substituting Eqs. (2), (3), and (9) into Eq. (1), we obtain the following:

$$
\left\{\begin{aligned}
\ddot{X}_{s q i} & =\frac{r_{c 1}}{M_{c} r_{b c}^{2}}\left(T_{D}-T_{L 2}\right. \\
& -\left(\sum_{i=1}^{N} K_{s q i}(t) f\left(X_{s q i}, b_{s q i}\right)\right. \\
& \left.\left.+\sum_{i=1}^{N} C_{s q i} \dot{X}_{s q i}\right) r_{b s} \cos \alpha\right) \\
& -\frac{1}{M_{q i}}\left(C_{s q i} \dot{X}_{s q i}+K_{s q i}(t)\right. \\
& +f\left(X_{s q i}, b_{s q i}\right)-C_{p i q i} \dot{X}_{p i q i} \\
& \left.-K_{p i q i}(t) f\left(X_{p i q i}, b_{p i q i}\right)\right)-\ddot{e}_{s q i}(t) \\
\ddot{X}_{p i q i} & =\frac{1}{M_{q i}}\left(C_{s q i} \dot{X}_{s q i}+K_{s q i}(t) f\left(X_{s q i}, b_{s q i}\right)\right. \\
& \left.-C_{p i q i} \dot{X}_{p i q i}-K_{p i q i}(t) f\left(X_{p i q i}, b_{p i q i}\right)\right) \\
& -\frac{1}{M_{p i} r_{b p i}}\left(\left(K_{p i q i}(t) f\left(X_{p i q i}, b_{p i q i}\right)\right.\right. \\
& \left.\left.+C_{p i q i} \dot{X}_{p i q i}\right) r_{b p i}-T_{L 1}\right) \\
& -\ddot{e}_{p i q i}(t)
\end{aligned}\right.
$$

where $M_{c}, M_{q i}, M_{p i}, M_{s}$ can be expressed as

$$
\begin{aligned}
M_{c} & =\left(J_{c}+\sum_{i=1}^{N}\left(m_{p i} r_{c 2}+m_{q i} r_{c 1}\right)\right) / r_{b c}^{2} ; \\
M_{q i} & =J_{q i} / r_{b q i}^{2} ; \\
M_{p i} & =J_{p i} / r_{b p i}^{2} ; \quad M_{s}=J_{s} / r_{b s}^{2} .
\end{aligned}
$$

In order to simplify the solutions of the equations and the analysis of the results, we use dimensionless variables, introducing the parameters

$$
\begin{aligned}
\omega_{n} & =\sqrt{K_{m s q i}\left(1 / M_{s}+1 / M_{c}\right)} ; \quad \bar{X}=X / b_{c} ; \\
\dot{\bar{X}} & =\dot{X} / \omega_{n} b_{c} ; \quad \ddot{\bar{X}}=\ddot{X} / \omega_{n}{ }^{2} b_{c} ; \\
\bar{b} & =b / b_{c} ; \quad \ddot{\bar{e}}=\ddot{e} / \omega_{n}{ }^{2} b_{c} ; \\
\Omega & =\omega / \omega_{n} ; \quad \tau=\omega_{n} t ;
\end{aligned}
$$

where $\tau$ is dimensionless time and $b_{c}$ is the nominal size of displacement. 
Substituting Eq. (12) into Eq. (11), we obtain the following:

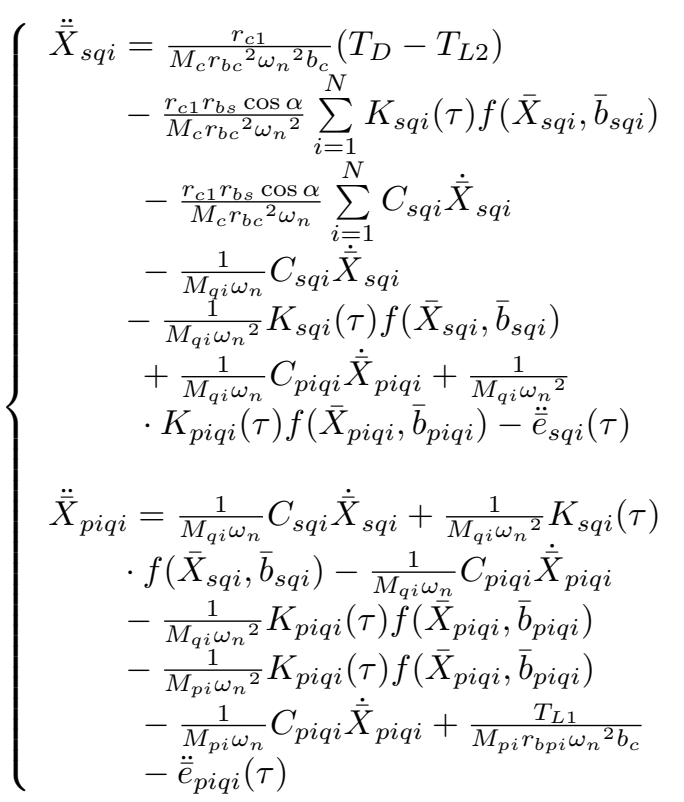

where $K_{\text {sqi }}(\tau), K_{\text {piqi }}(\tau), \bar{e}_{s q i}(\tau), \bar{e}_{\text {piqi }}(\tau)$ can be represented by

$$
\begin{gathered}
\left\{\begin{array}{l}
K_{s q i}(\tau)=K_{m s q i}+K_{a s q i} \sin \left(\Omega \tau+\varphi_{s q i}\right) \\
K_{\text {piqi }}(\tau)=K_{\text {mpiqi }}+K_{\text {apiqi }} \sin \left(\Omega \tau+\varphi_{\text {piqi }}\right)
\end{array}\right. \\
\left\{\begin{array}{l}
\bar{e}_{s q i}(\tau)=\frac{E_{s q i}}{b_{c}} \sin \left(\Omega \tau+\phi_{s q i}\right) \\
\bar{e}_{p i q i}(\tau)=\frac{E_{p i q i}}{b_{c}} \sin \left(\Omega \tau+\phi_{\text {piqi }}\right)
\end{array}\right.
\end{gathered}
$$

\section{THE STEADY-STATE RESPONSE OF THE SYSTEM}

In order to investigate the vibration characteristics of the planetary gear processing device, this paper uses a set of basic parameters: $m=3.0 \mathrm{~mm}, \alpha=20^{\circ}, E_{s q i}=E_{\text {piqi }}=$ $10 \mu \mathrm{m}, \varphi_{\text {sqi }}=\varphi_{\text {piqi }}=0, \phi_{\text {sqi }}=\phi_{\text {piqi }}=0, z_{s}=40$, $z_{q i}=30, z_{p i}=40, N=3, b_{c}=10 \mu \mathrm{m}, B=15 \mathrm{~mm}$, $b_{s q i}=b_{\text {piqi }}=50 \mu \mathrm{m}, K_{m s q i}=0.8256 \mathrm{GN} / \mathrm{m}, K_{m p i q i}=$ $1.06 \mathrm{GN} / \mathrm{m}, K_{\text {asqi }}=K_{\text {apiqi }}=0.2 \mathrm{GN} / \mathrm{m}, T_{D}=1100 \mathrm{Nm}$, $T_{L 1}=100 \mathrm{Nm}$, and $T_{L 2}=1000 \mathrm{Nm}$.

This paper uses a numerical integration algorithm to solve the nonlinear Eq. (13) with the four order Runge-Kutta method, and investigates the steady-state responses of the planetary gear system. The bifurcation diagram of the system with the non-dimensional speed is shown in Fig. 4.

Figure 4 shows that the system is stable excepting the critical speed $\Omega=0.75$ to 2.3 , and the region is also called the chaotic region. Simultaneously, when the non-dimensional speed is at $\Omega=0.75$ to 2.3 , the non-dimensional displacement $\bar{X}_{s} p i$ has a magnitude of nearly 2 to 4 .

Figure 5 shows that the bifurcation diagram of the vibration system changes with the dimensionless speed $\Omega$ when the damping ratios are at $\xi_{1}=\xi_{2}=0.1$. The bifurcation diagram is different from the diagram in Fig. 4. The amplitude range of the non-dimensional displacement shows a sharp decline compared to the system in Fig. 5. In addition, the vibration system only passes through a short chaotic region with the speed $\Omega$ form 1 to 2, and the displacement amplitudes in Fig. 4 change

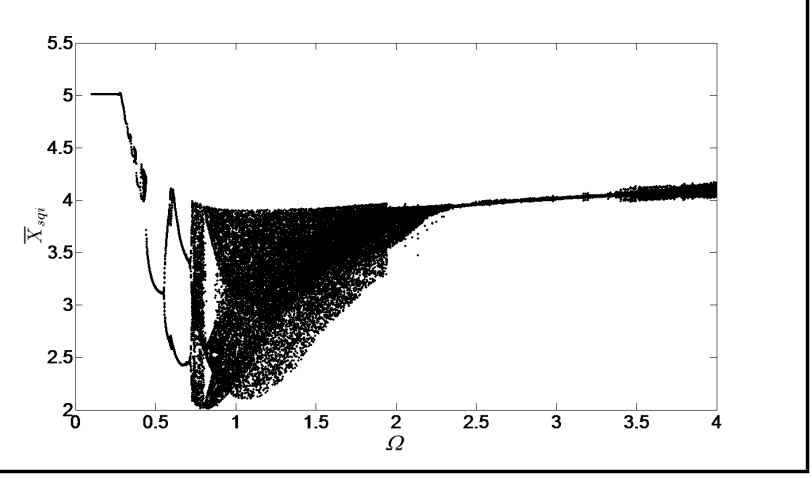

Figure 4. Bifurcation diagram of the system with non-dimensional planetary speed $\Omega\left(\xi_{1}=\xi_{2}=0.05\right)$.

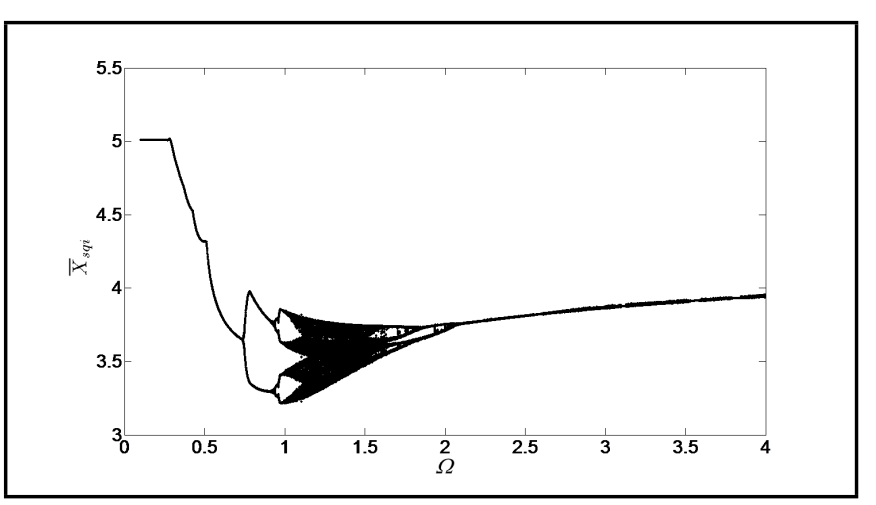

Figure 5. Bifurcation diagram of the system with non-dimensional planetary speed $\Omega\left(\xi_{1}=\xi_{2}=0.1\right)$.

more than those in Fig. 5. Simultaneously, the vibration state is in the stage of aperiodic motion. So, this paper pays more attention to the vibration with $\xi_{1}=\xi_{2}=0.05$.

Figure 6 shows that the vibration of the system is a harmonic response by the excitation for $\Omega=0.2$. The time histories are sinusoidal waves, and the phase plot shows an ellipse. Whats more, the Poincar map is a single scatter, and the Fourier spectra shows that the frequency occurs at $\Omega$. The results indicates that the system is stable.

Figure 7 shows that the vibration of the system is a nonharmonic response by the excitation for $\Omega=0.4$. The time histories are nearly single periodic motions, which consist of different sinusoidal waves, but the phase plot does not show an ellipse. The Poincar map has many scatters that are close to each other. The Fourier spectra shows that the frequency occurs at $k \Omega$ ( $k$ is a positive integer). The results indicate that the system response is a super-harmonic response.

Figure 8 indicates that the vibration of the system is a $2 \mathrm{~T}$ periodic harmonic response by the excitation for $\Omega=0.56$. The time histories are nearly two periodic motions, which consist of sinusoidal waves, and the phase plot shows two ellipses. The Poincar map has two scatters that are not close to each other. The Fourier spectra shows that the frequency occurs at . The results indicate that the system response is stable.

Figure 9 shows that the system creates a 4T-periodic harmonic response by the excitation for $\Omega=0.72$. The time histories are nearly four periodic motions which consist of sinusoidal waves, and the phase plot shows four quasi ellipses. The Poincar map has four scatters that are not close to each other. The Fourier spectra shows that the frequency occurs at $\Omega$. The 


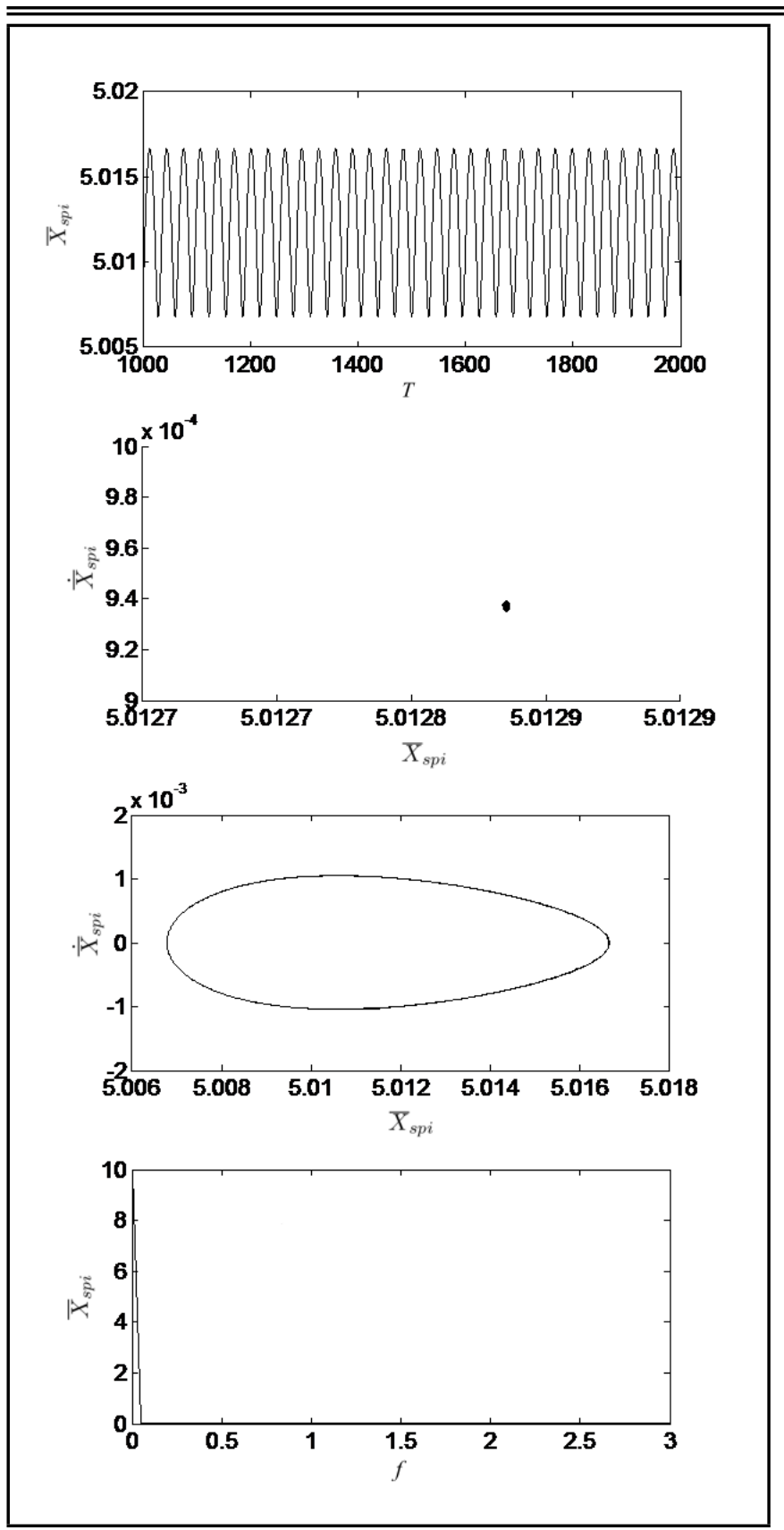

Figure 6. Harmonic response $(\Omega=0.2)$. (a) Time histories; (b) Poincaré map; (c) Phase plant plot; (d) Fourier spectrum.

results indicate that the system response is still stable.

Figure 10 shows that the vibration of the system is a quasiperiodic response by the excitation for $\Omega=0.74$. The time histories are quasi-periodic motions which consist of different kinds of sinusoidal waves, and the phase plot consists of many analogical ellipses. The Poincar map has many scatters, which form the instability attractor. The results indicate that the system response is in a critical state, and the results reveal that the device is easy to work in the next state.

Figure 11 shows that the system creates the chaotic responce by the excitation for $\Omega=1.5$. The time histories are not periodic motions which consist of different kinds of waves, and the phase plot consists of many different ellipses or spiral lines. The Poincar map has many scatters, which form the instability attractor and irregular shape. The results indicate that the system response is not stable, and the device is easily dam-

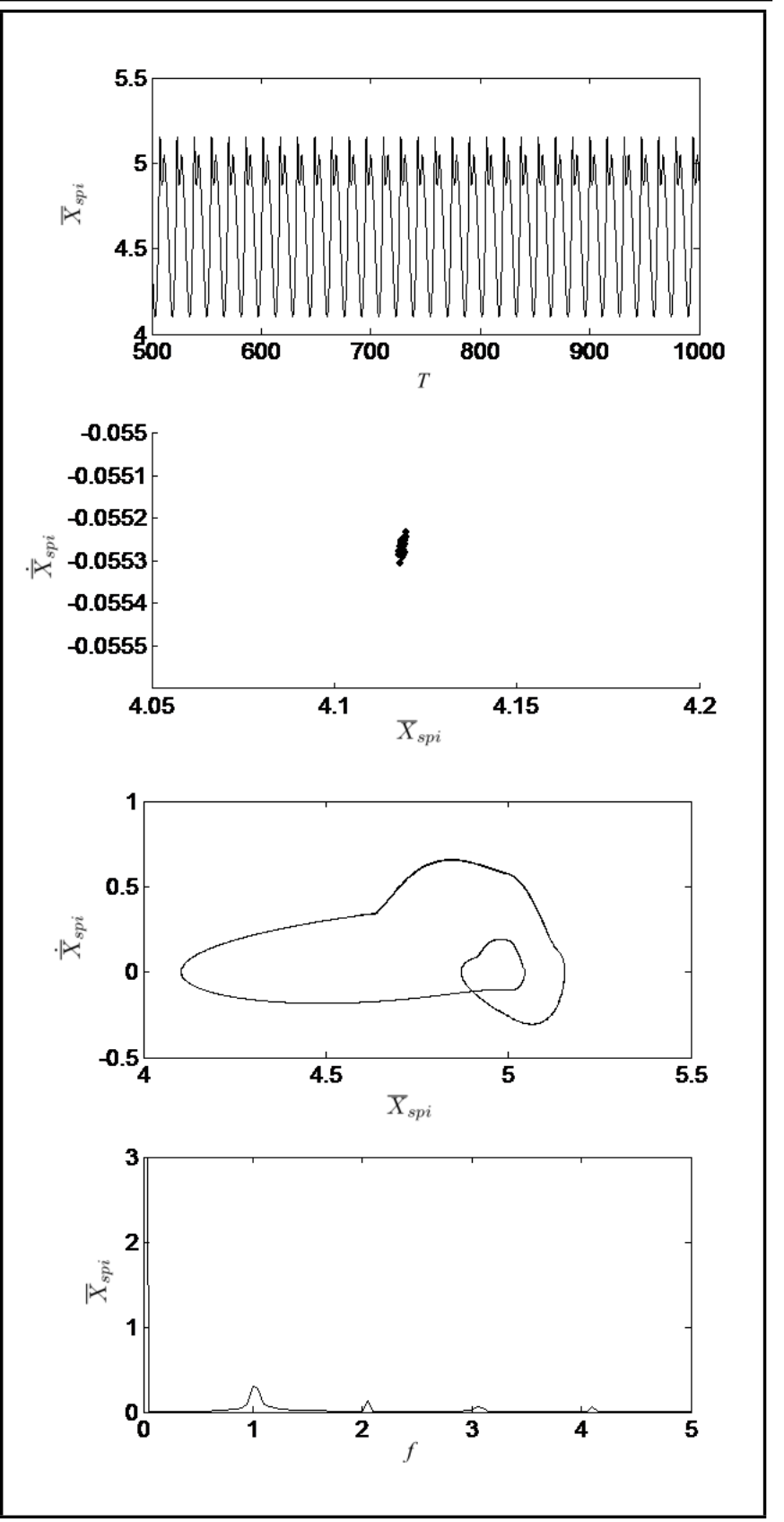

Figure 7. Non-harmonic response $(\Omega=0.4)$. (a) Time histories; (b) Poincaré map; (c) Phase plant plot; (d) Fourier spectrum.

aged. That is to say, the planetary gear processing device will be damaged in the form of fatigue.

Figure 12 shows that the vibration of the system returns to the harmonic response by the excitation for $\Omega=3$. The time histories are still periodic motions which consist of sinusoidal waves, and the phase plot consists of only one ellipse. The Poincar map has many scatters, which are close to each other. The results illstrate that the system response is stable, and the planetary gear processing device will vibrate in the form of harmonic motion.

\section{CONCLUSIONS}

This paper analyszed the planetary gear processing device and established the nonlinear dynamic model. This vibration model considered errors of transmission, time varying meshing stiffness, and gear backlashes. Then, the solution of the dimen- 


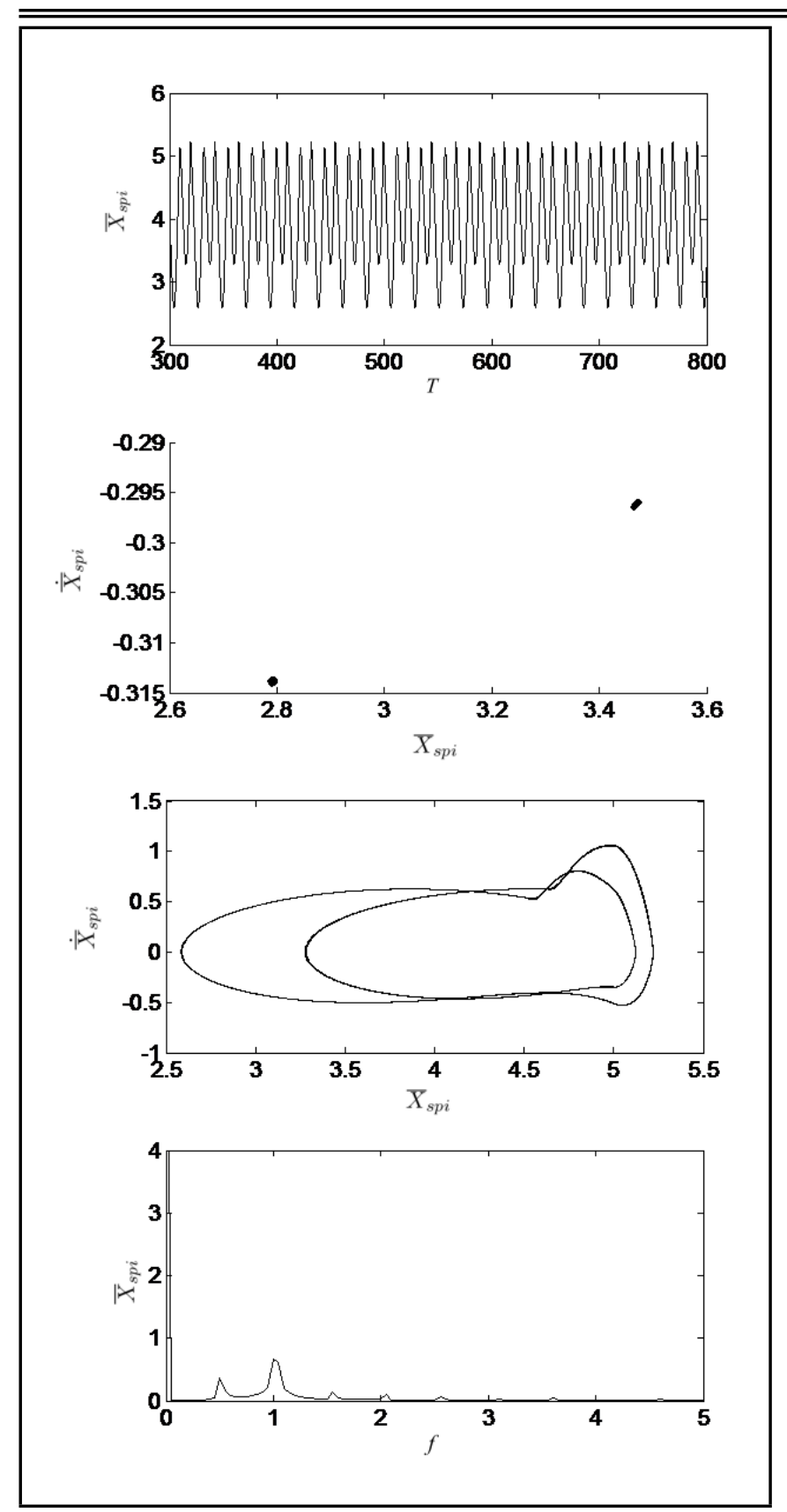

Figure 8. The 2T-periodic harmonic response $(\Omega=0.56)$. (a) Time histories; (b) Poincaré map; (c) Phase plant plot; (d) Fourier spectrum.

sionless equations of the system was carried out by using the method of numerical integration. By comparison to Poincar maps and bifurcation diagrams, the vibration properties of the planetary gear system were investigated, and the following results were found:

1. The planetary gear train system with translational motion has abundant bifurcation characteristics because of the complex influences of many nonlinear factors. The results reveal that the motion state of the system will change into chaos in the way of crisis as the dimensionless speed increases.

2. The system will be in the chaotic state when the dimensionless speed is increased to a certain value range. Therefore, the system should avoid these critical regions for reducing fatigue-failure of the processing device.
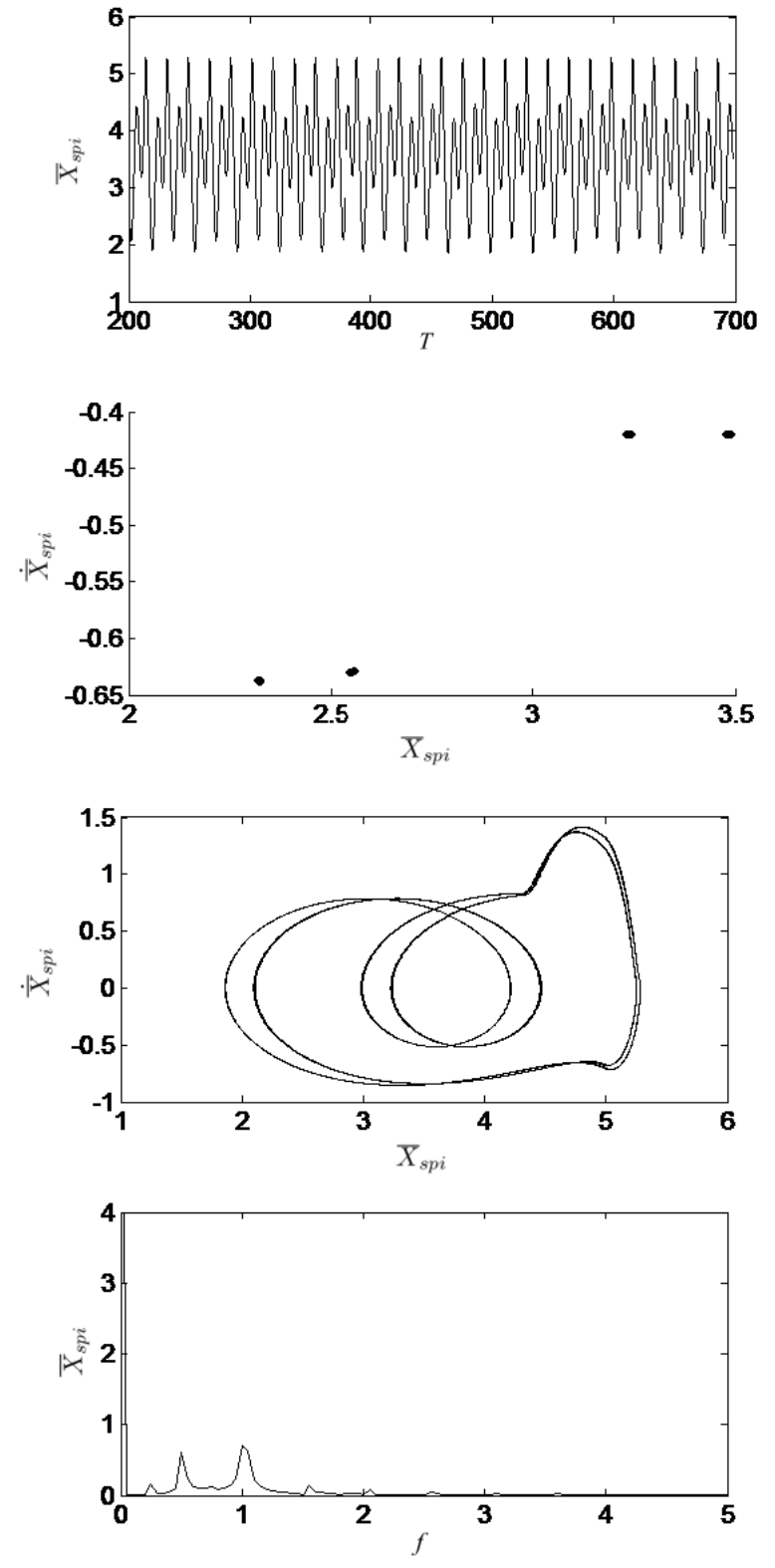

Figure 9. The 4T-periodic harmonic response $(\Omega=0.72)$. (a) Time histories; (b) Poincaré map; (c) Phase plant plot; (d) Fourier spectrum.

3. The time histories, phase plane plots, Poincar maps, and Fourier spectras prove the planetary gear train system has a harmonic response, a 2T-period harmonic response, a 4T-period harmonic response, a quasi-harmonic response, and a chaotic response, but the chaotic state is not stable and should be avoided.

4. The damping ratio can influence the vibration amplitudes greatly, and it should be increased when the processing device is designed. Also, the damping ratio can easily influence the bifurcation characteristics.

\section{ACKNOWLEDGMENTS}

This paper was supported by National Natural Science Foundation of China (Grant No.51375320). The authors would like to acknowledge their financial support. 


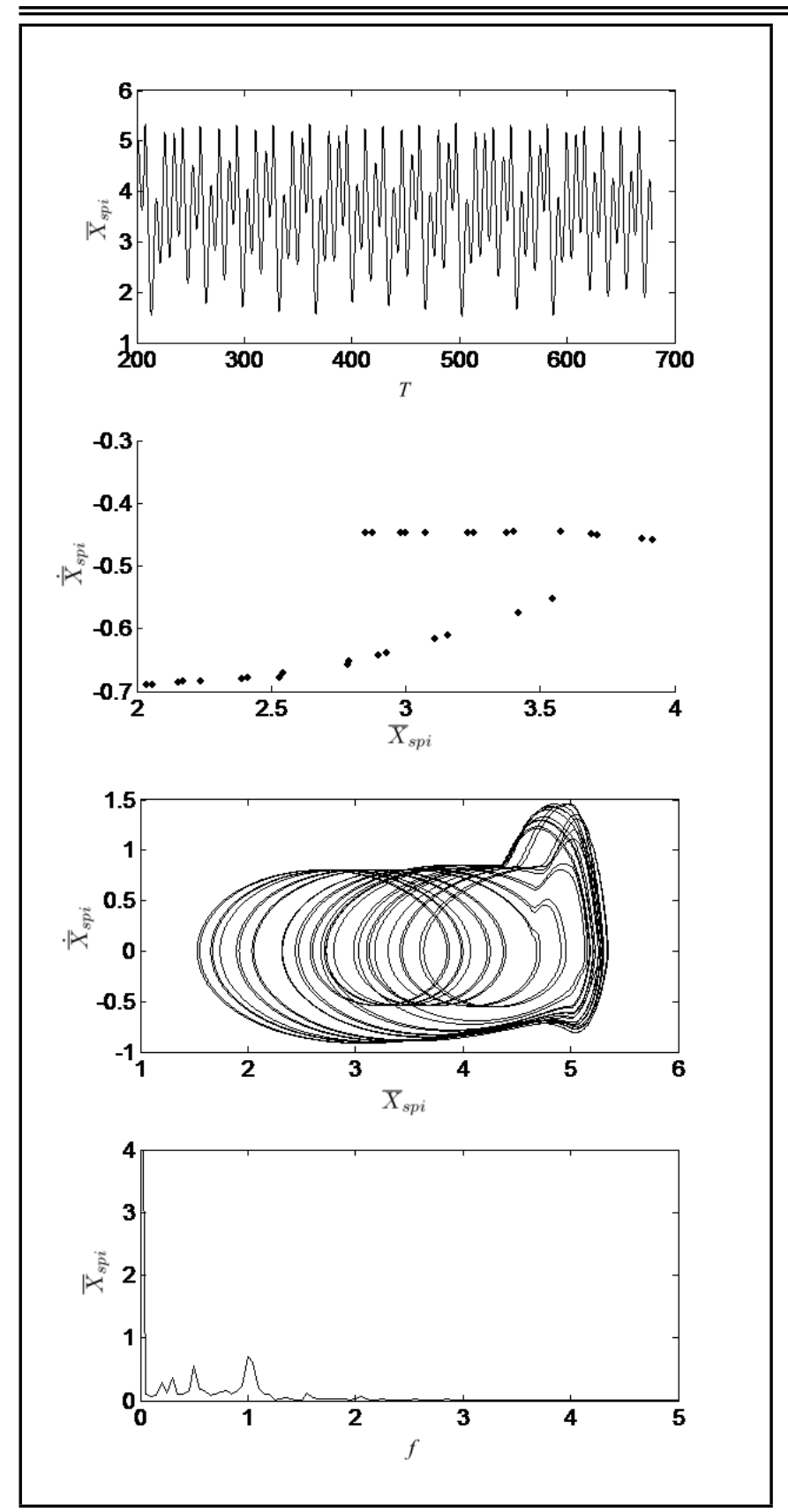

Figure 10. The quasi periodic response $(\Omega=0.74)$. (a) Time histories; (b) Poincaré map; (c) Phase plant plot; (d) Fourier spectrum.

\section{REFERENCES}

1 Chen, Z. and Shao, Y. Dynamic simulation of planetary gear with tooth root crack in ring gear, Eng. Fail. Anal., 31, 818, (2013). http://dx.doi.org/10.1016/j.engfailanal.2013.01.012

2 Song, A., Wu, W., Gao, S., and Gao, W. The ideal geometry parameters of arch cylindrical gear and its process method, J. Shanghai Jiaotong U., 44 (12), 17351740, (2010).

3 Velex, P. and Flamand, L. Dynamic response of planetary trains to mesh parametric excitations, J. Mech. Des., 118, 714, (1996). http://dx.doi.org/10.1115/1.2826860

4 Kahraman, A. and Blankenship, G. W. Experiments on nonlinear dynamic behavior of an oscillator with clearance and
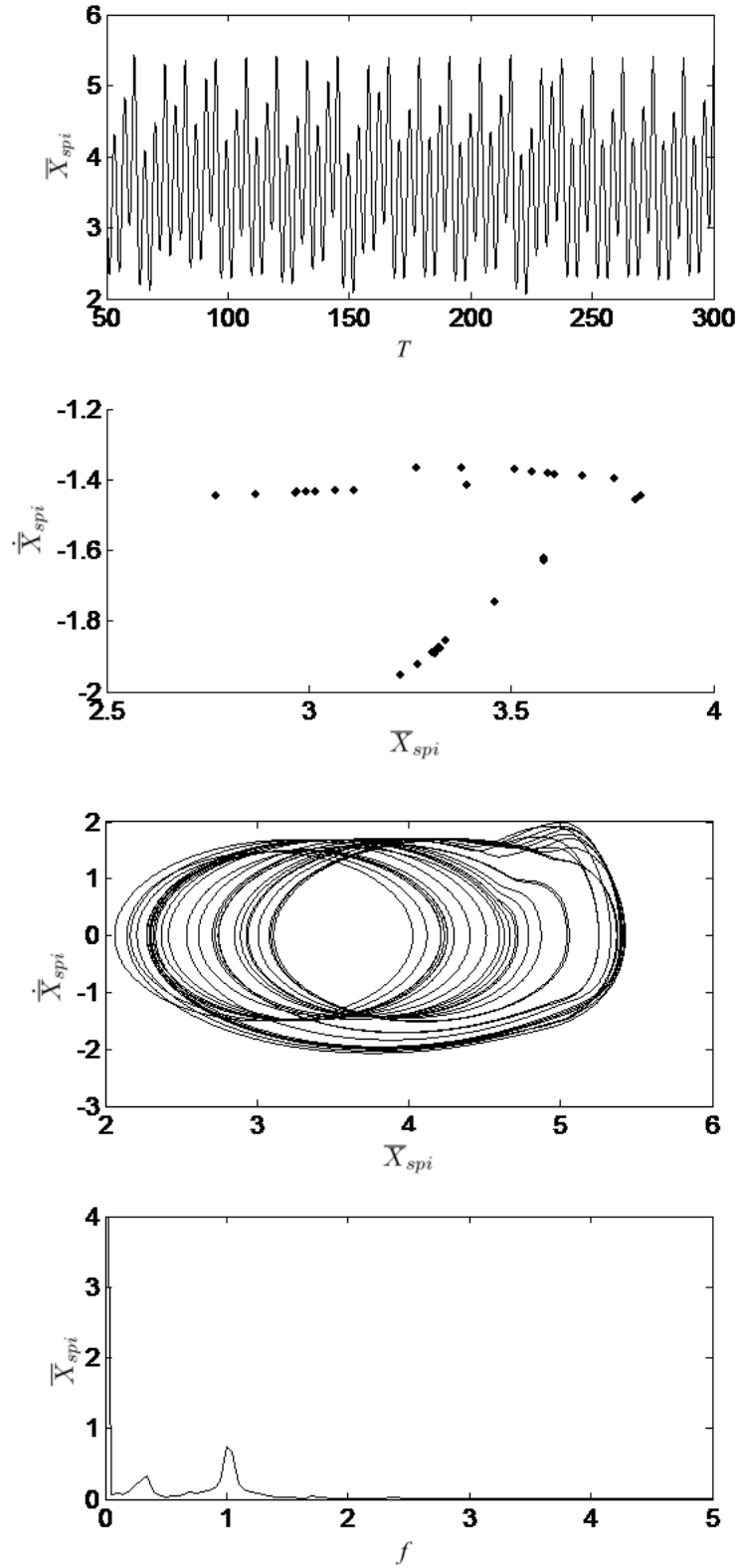

Figure 11. The chaotic resonance $(\Omega=1.5)$. (a) Time histories; (b) Poincaré map; (c) Phase plant plot; (d) Fourier spectrum.

periodically time-varying parameters, J. Appl. Mech., 64, 217226, (1997). http://dx.doi.org/10.1115/1.2787276

5 Lin, J. and Parker, R. G. Planetary gear parametric instability caused by mesh stiffness variation, J. Sound Vib., 249 (1), 129145, (2002). http://dx.doi.org/10.1006/jsvi.2001.3848

6 Kahraman, A. Natural modes of planetary gear trains, J. Sound Vib., 173 (1), 125130, (1994). http://dx.doi.org/10.1006/jsvi.1994.1222

7 Parker, R. G. and $\mathrm{Wu}, \mathrm{X}$. Vibration modes of planetary gears with unequally spaced planets and an elastic ring gear, J. Sound Vib., 329, 22652275, (2010). http://dx.doi.org/10.1016/j.jsv.2009.12.023

8 Ericson, T. M. and Parker, R. G. Experimen- 


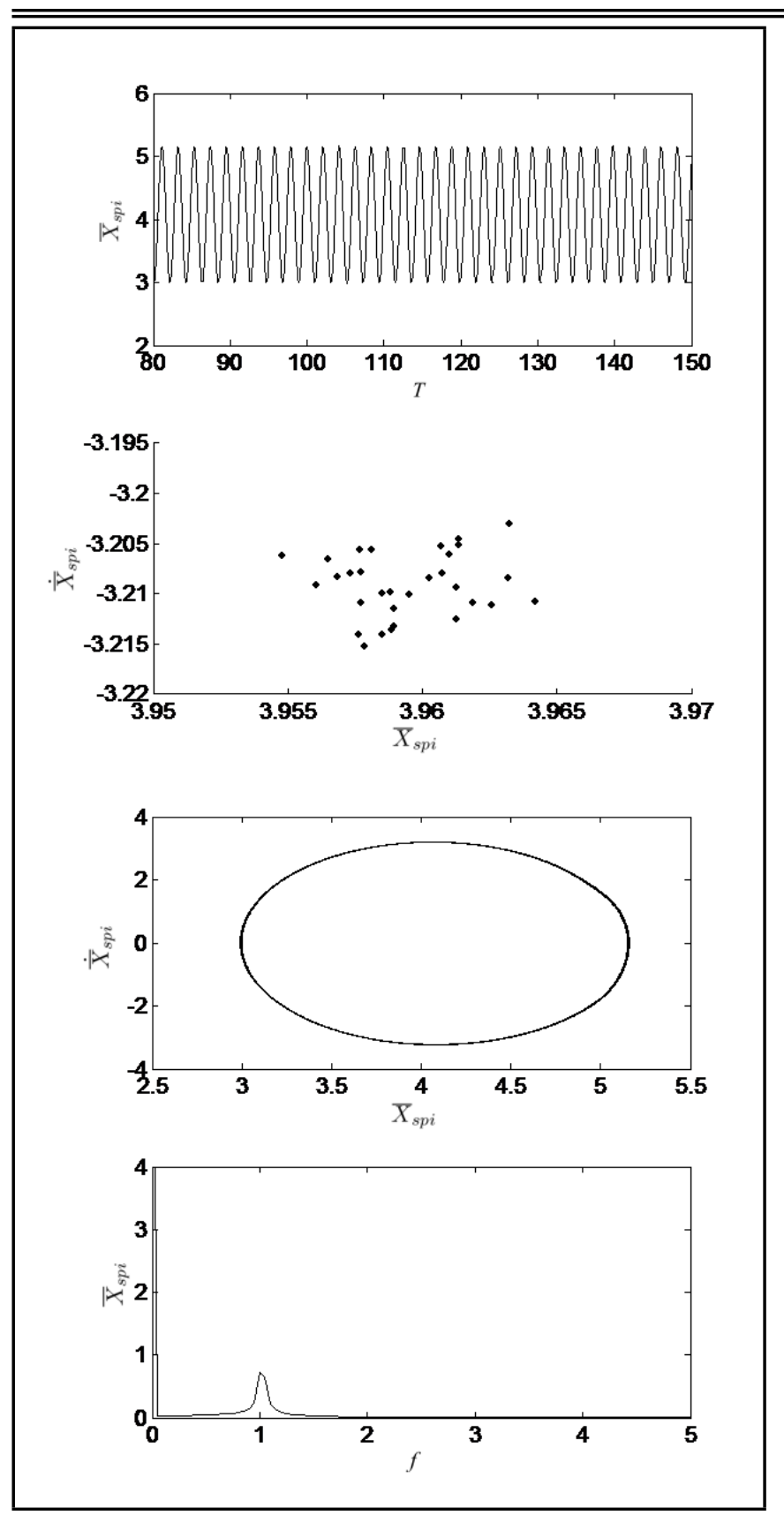

Figure 12. The harmonic response $(\Omega=3)$. (a) Time histories; (b) Poincaré map; (c) Phase plant plot; (d) Fourier spectrum.

tal measurement of the effects of torque on the dynamic behavior and system parameters of planetary gears, Mech. Mach. Theory, 74, 370389, (2014). http://dx.doi.org/10.1016/j.mechmachtheory.2013.12.018

9 Ericson, T. M. and Parker, R. G. Planetary gear modal vibration experiments and correlation against lumped-parameter and finite element models, J. Sound Vib., 332, 23502375, (2013). http://dx.doi.org/10.1016/j.jsv.2012.11.004

10 Chaari, F., Fakhfakh, T., Hbaieb, R., Louati, J., and Haddar, M. Influence of manufacturing errors on the dynamical behavior of planetary gear, Int. J. Adv. Manuf. Tech., 27, 738746, (2006). http://dx.doi.org/10.1007/s00170-0042240-2
11 Lin, J. and Parker, R. G. Structured vibration characteristics of planetary gears with unequally spaced planets, J. Sound Vib., 233 (5), 921928, (2000). http://dx.doi.org/10.1006/jsvi.1999.2581

12 Chen, Z. G., Shao, Y. M., and Lim, T. C. Non-linear dynamic simulation of gear response under the idling condition, Int. J. Automot. Techn., 13 (4), 541552, (2012). http://dx.doi.org/10.1007/s12239-012-0052-1

13 Xihui, L., Zuo, M. J., and Pandey, M. Analytically evaluating the influence of crack on the mesh stiffness of a planetary gear set, Mech. Mach. Theory, 76, 2038, (2014). http://dx.doi.org/10.1016/j.mechmachtheory.2014.02.001

14 Li, S., Wu, Q., and Zhang, Z. Bifurcation and chaos analysis of multistage planetary gear train, Nonlinear Dynam., 75 (12), 217233, (2014). http://dx.doi.org/10.1007/s11071$013-1060-\mathrm{z}$

15 Kahraman, A. Free torsional vibration characteristics of compound planetary gear sets, Mech. Mach. Theory, 36, 953971, (2001). http://dx.doi.org/10.1016/s0094114x(01)00033-7

16 Parker, R. G. A physical explanation for the effectiveness of planet phasing to suppress planetary gear vibration, J. Sound Vib., 236 (4), 561573, (2000). http://dx.doi.org/10.1006/jsvi.1999.2859

17 Lin, J. and Parker, R. G. Sensitivity of planetary gear natural frequencies and vibration modes to model parameters, J. Sound Vib., 228 (1), 109128, (1999). http://dx.doi.org/10.1006/jsvi.1999.2398

18 Lin, J. and Parker, R. G. Analytical characterization of the unique properties of planetary gear free vibration, J. Vib. Acoust., 121 (3), 316321, (1999). http://dx.doi.org/10.1115/1.2893982

19 Ambarisha, V. K. and Parker, R. G. Nonlinear dynamics of planetary gears using analytical and finite element models, J. Sound Vib., 302, 577595, (2007). http://dx.doi.org/10.1016/j.jsv.2006.11.028

20 Zhimin, S., Linhong, J., and Yunwen, S. Nonlinear dynamics of $2 \mathrm{~K}-\mathrm{H}$ planetary gear train, J. Tsinghua U. (Science \& Technology), 43 (5), 636639, (2003).

$21 \mathrm{Li}, \mathrm{T}$. Nonlinear torsional vibration modeling and bifurcation characteristic study of a planetary gear train, J. Mech. Eng., 47 (21), 7683, (2011). http://dx.doi.org/10.3901/jme.2011.21.076

22 Runfang, L. and Jiangjun, W. Dynamics of gear system: Vibration, shock and noise, Science Press, Beijing, (1997). 